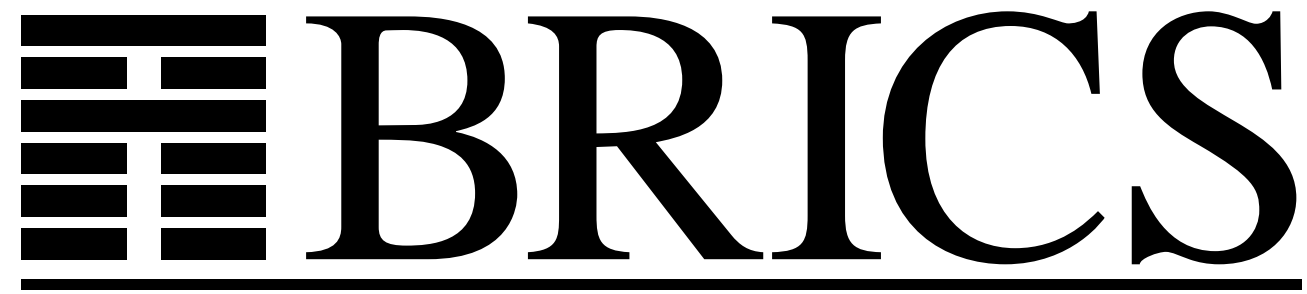

Basic Research in Computer Science

\title{
A Note on Frame Distributions
}

Anders Kock

Gonzalo E. Reyes 
Copyright (c) 1997, $\quad$ BRICS, Department of Computer Science University of Aarhus. All rights reserved.

Reproduction of all or part of this work is permitted for educational or research use on condition that this copyright notice is included in any copy.

See back inner page for a list of recent BRICS Report Series publications. Copies may be obtained by contacting:

\author{
BRICS \\ Department of Computer Science \\ University of Aarhus \\ Ny Munkegade, building 540 \\ DK-8000 Aarhus C \\ Denmark \\ Telephone: +4589423360 \\ Telefax: $\quad+4589423255$ \\ Internet: BRICS@brics.dk
}

BRICS publications are in general accessible through the World Wide Web and anonymous FTP through these URLs:

http://www.brics.dk

ftp: //ftp.brics.dk

This document in subdirectory RS/97/33/ 


\title{
A Note on Frame Distributions
}

\author{
Anders Kock and Gonzalo E. Reyes
}

\begin{abstract}
In the context of constructive locale or frame theory (locale theory over a fixed base locale), we study some aspects of 'frame distributions', meaning sup preserving maps from a frame to the base frame. We derive a relationship between results of Jibladze-Johnstone and Bunge-Funk, and also descriptions in distribution terms, as well as in double negation terms, of the 'interior of closure' operator on open parts of a locale.
\end{abstract}

\section{Introduction}

This paper grew out of an interest in studying constructive locale theory, and thus continues the tradition from [6], [5], [3], [8], [1], and many others ${ }^{1}$.

More precisely, we study locales in a topos, and in particular, locales over a given base locale; so our study comprises what [5] calls fibrewise notions, like fibrewise dense, and fibrewise closed. The methods are of algebraic nature, with emphasis on frames, nuclei, and lattice theory in general.

The paper is divided into three sections, all three of elementary lattice theoretic character. In the first, we derive the Jibladze-Johnhnstone Theorem (characterizing relatively closed sublocales) by analyzing a certain pair of adjoint functors. We use this, in the second section, to derive a relationship between certain "intensive" and "extensive" quantities (in the sense of Lawvere [10]) on an open locale; the extensive quantities in question being certain "frame distributions", suggested by Lawvere [9] [10], and studied by Bunge and Funk in [1]. We give an alternative proof of their result: identifying these frame-distributions on a locale $M$ with certain sublocales of $M$.

\footnotetext{
${ }^{1}$ A preliminary version "Frame distributions and support" was made available on the internet already in January 1996 (as announced on the Categories Mailing List) The present version is identical to that one, except for some omissions and slight reformulations.
} 
In the third section we prove that three closure operators on an open frame coincide; one of these derives from the adjoint pair relating opens and frame distributions, the other from interior and relative closure on sublocales, and the third being a generalized double negation nucleus.

We would like to thank Marta Bunge and Steve Vickers for fruitful discussions on some of the topics treated here. The research of the second author was partly supported by a grant from the National Research and Engineering Research Council of Canada.

\section{The Jibladze-Johnstone correspondence}

The title of this section refers to a bijection between "fibrewise closed" nuclei on a frame $A$, relative to a fixed base frame $\phi: B \rightarrow A$, and a certain equationally described class of maps $B \rightarrow A$, called the $B$-nuclei on $A$. We shall give an alternative, elementary, description of this correspondence, deriving it from an adjointness, (and using a "generalized double negation nucleus").

Let $A$ be an arbitrary frame, and $\phi: X \rightarrow A$ a family of elements in $A$ ( $X$ being an arbitrary set; in the Jibladze-Johnstone case [3], $X$ would be the base frame $B$ and $\phi$ would be a frame map). We shall keep $X$ fixed in what follows, and often omit the map $\phi$ from the notation, i.e. consider $X$ as a subset of the frame $A$. Let as usual $N A$ denote the frame of nuclei on $A$, under the pointwise order (so $N A$ is the dual lattice of the lattice of sublocales of the locale corresponding to $A$ ). Also, let $A^{X}$ be the frame of all maps from $X$ to $A$ (with pointwise frame structure). We have a map $\Phi: N A \rightarrow A^{X}$, namely restriction along $\phi$ (so $\phi$ takes a nucleus $j: A \rightarrow A$ to the map $j \circ \phi: X \rightarrow A$ ). Since infima are computed pointwise in $N A$ as well as in $A^{X}$, it follows that $\Phi$ preserves infima, and thus has a left adjoint $\Psi$. The fixpoint sets of the two composites $\Phi \circ \Psi$ and $\Psi \circ \Phi$ are therefore isomorphic, via $\Phi$ and $\Psi$. We shall in fact prove that this isomorphism is the correspondence of [3].

We first prove that the formula of Johnstone and Jibladze (Lemma 1.1 of [3]) provides the left adjoint $\Psi$ of the "restriction" map $\Phi$ :

Proposition 1 If $k: X \rightarrow A$ is any map, $\Psi(k)$ is the nucleus

$$
\bigvee_{x \in X}\{c(k(x)) \wedge o(x)\}
$$


where the join is taken in the frame $N A$, and where $c(k(x))$ denotes the closed nucleus $k(x) \vee-$ on $A$ and $o(x)$ denotes the open nucleus $x \rightarrow-$ on $A$.

Proof. We prove the two inequalities

$$
\Psi(\Phi(j)) \leq j
$$

for any nucleus $j$ on $A$, and

$$
k \leq \Phi(\Psi(k))
$$

for any map $k: X \rightarrow A$. As for the first, this amounts to proving

$$
\bigvee\{c(j(x)) \wedge o(x)\} \leq j
$$

so for each $x \in X$, we should prove $c(j(x)) \wedge o(x) \leq j$, i.e. for each $a \in A$, we should prove $(j(x) \vee a) \wedge(x \rightarrow a) \leq j(a)$. Using distributivity of $\wedge$ over $\vee$, this amount to proving the two inequalities $j(x) \wedge(x \rightarrow a) \leq j(a)$ and $a \wedge(x \rightarrow a) \leq j(a)$. The former follows from $(x \rightarrow a) \leq(j(x) \rightarrow j(a))$, and the second from $a \wedge(x \rightarrow a)=a$ and $a \leq j(a)$.

For the second inequality, we should prove for each $y \in X$ that

$$
k(y) \leq\left(\bigvee_{x \in X}\{c(k(x)) \wedge o(x)\}\right)(y)
$$

it suffices to prove $k(y) \leq(c(k(y)) \wedge o(y))(y)$, i.e. $k(y) \leq(k(y) \vee y) \wedge(y \rightarrow y)$, which is clear, without any assumptions on $k$.

By the adjointness $\Psi \vdash \Phi$, it follows that the fixpoints for $\Psi \circ \Phi: N A \rightarrow$ $N A$ consist of those elements $j$ which are minimal in $\Phi^{-1}(\Phi(j))$, i.e. consists of nuclei $j$ which are smallest among those with a given restriction along $X \rightarrow A$. This is precisely the definition of $j$ being closed relative to $X \rightarrow A$ ("fibrewise closed" in the terminology of [3]). This identifies the fixpoint lattice of $\Psi \circ \Phi$ as the relatively closed nuclei on $A$ (relative to $X \rightarrow A$ ). (And the operator $\Psi \circ \Phi$ is closure-relative-to- $X$.)

Theorem 1 A map $k: X \rightarrow A$ is a fixpoint for $\Phi \circ \Psi: A^{X} \rightarrow A^{X}$ if and only if $k$ satisfies the equation

$$
x \rightarrow k(y)=k(x) \rightarrow k(y)
$$

for all $x, y \in X$. 
(Recall that $x \rightarrow k(y)$ in full notation would be $\phi(x) \rightarrow k(y)$; the equation of the theorem is of course the definition in [3] of the notion of an $X$-nucleus. So in some sense, our result gives a "proof" of that definition, i.e. derives it from an adjointness.)

Proof. If $j \in N A$ is a nucleus, then its restriction along $\phi: X \rightarrow A$ satisfies the equation ( 1$)$; this is essentially Corollary 3.2 in [3], and is anyway an immediate consequence of the law $a \rightarrow j(b)=j(a) \rightarrow j(b)$ that holds for all nuclei $j$. Since any fixpoint of $\Phi \circ \Psi$ is in the image of $\Phi$, this proves that fixpoints $k$ satisfy the equation. Conversely, assume that $k: X \rightarrow A$ satisfies the equation ( 1$)$. To prove $k=\Phi(\Psi(k))$, it suffices to prove

$$
\Phi(\Psi(k) \leq k,
$$

since the adjointness takes care of the other inequality. Let us, for $x \in X$, denote the nucleus $c(k(x)) \wedge o(x)$ by $j_{x}$; so that we should show $\bigvee_{x} j_{x} \leq k$. The join here is not formed pointwise; the trick is to find a generalized double negation nucleus which is an upper bound for the constituents.

Specifically, we first prove that (1) implies that, for every $x \in X, j_{x} \circ k=$ $k$. We have in fact, for $x, y \in X$ that

$$
\begin{aligned}
j_{x}(k(y))= & (c(k(x)) \wedge o(x))(k(y)) \\
& =(k(x) \vee k(y)) \wedge(x \rightarrow k(y)) \\
& =k(x) \wedge(x \rightarrow k(y)) \vee(k(y) \wedge(x \rightarrow k(y))) \\
& =k(x) \wedge(x \rightarrow k(y)) \vee k(y) \\
& =k(x) \wedge(k(x) \rightarrow k(y)) \vee k(y) \text { (using equation }(1)) \\
& =(k(x) \wedge k(y)) \vee k(y)=k(y) .
\end{aligned}
$$

Using the law $j a \wedge(a \rightarrow b) \leq j b$ which holds for any nucleus $j$, we therefore have, for all $x, y$ in $X$ and $a$ in $\mathrm{A}$,

$$
j_{x}(a) \wedge(a \rightarrow k(y)) \leq j_{x}(k(y))=k(y)
$$

and so $j_{x}(a) \leq(a \rightarrow k(y)) \rightarrow k(y)$. Thus we have for each $x$ that

$$
j_{x} \leq \bigwedge_{y \in X}((-) \rightarrow k(y)) \rightarrow k(y) .
$$

Since the right hand side here is a (generalized double negation) nucleus, we get in $N A$ that 


$$
\bigvee_{x \in X} j_{x} \leq \bigwedge_{y \in X}((-) \rightarrow k(y)) \rightarrow k(y) .
$$

Applying the two nuclei appearing here on an arbitrary $z \in X$, we get

$$
\left(\bigvee_{x \in X} j_{x}\right)(z) \leq \bigwedge_{y \in X}(z \rightarrow k(y)) \rightarrow k(y) \leq(z \rightarrow k(z)) \rightarrow k(z)=k(z)
$$

(the last by $\phi \leq k$ which follows from the consequence $z \rightarrow k(z)=k(z) \rightarrow$ $k(z)=1$ of (1). This proves the desired inequality and thus the Theorem.

\section{Distributions and support}

We consider in this section frames $A$ in an arbitrary topos (of which we talk as if it were the category of sets). We also consider the frame $\Omega$ of "truth values" in the topos. It is the initial frame, and the unique frame map $\Omega \rightarrow A$ we denote by $\phi$. We are then in the situation of the previous section, with $X=\Omega$, Since the $x \in X$ now are truth values, we find it more natural to denote them $\lambda, \lambda^{\prime}$, etc. (Top and bottom will be denoted by 1 and 0 , though, not 'true' and 'false'.)

We collect in the following two Propositions some, probably well known, facts from intuitionistic lattice theory. First

Proposition 2 The (unique) frame map $\phi: \Omega \rightarrow A$ is the smallest among all maps (order preserving or not) that preserve 1 .

Proof. Let $k: \Omega \rightarrow A$ preserve 1. Since in $\Omega, \lambda=\bigvee\{1 \mid \lambda\}$ and $\phi$ preserves suprema and 1 , we get $\phi(\lambda)=\bigvee\left\{1_{A} \mid \lambda\right\}$. To prove $\bigvee\left\{1_{A} \mid \lambda\right\} \leq$ $k(\lambda)$, it suffices to prove $1_{A} \leq k(\lambda)$ assuming that $\lambda$ holds, i.e. under the assumption that $\lambda=1$, which is clear since $k(1)=1_{A}$ by assumption.

Proposition 3 Let $\mu: A \rightarrow \Omega$ be sup preserving. Then we have the "Frobenius law"

$$
\mu(a \wedge \phi(\lambda))=\mu(a) \wedge \lambda
$$

for all $a \in A$ and $\lambda \in \Omega$. 
(The Proposition holds even when $A$ is just assumed to be a sup lattice, and $\phi$ preserves sup and 1.)

Proof. We note that $\mu \circ \phi \leq$ identity $_{\Omega}$. For, $\Omega$ is the free sup lattice on one generator 1 , and certainly $\mu(\phi(1)) \leq 1$. The proof of the Proposition is now obvious: viewing the two sides of the Frobenius law as defining maps $A \times \Omega \rightarrow \Omega$, we just have to see that the subobjects of $A \times \Omega$ classified by the two sides are equal, i.e., assume the left hand side (for a given $a, \lambda$ ) is 1 , prove that so is the right hand side, and vice versa, which is trivial, using $\mu(\phi(\lambda)) \leq \lambda$.

Proposition 4 Assume $k: \Omega \rightarrow A$ is inf-preserving, or equivalently, that is has a left adjoint. Then it is an $\Omega$-nucleus, i.e satisfies the equation (1), and hence, by Theorem 1, is fixed for the construction $\Phi \circ \Psi$.

Proof. For $\lambda$ and $\lambda^{\prime}$ in $\Omega$, we have must prove

$$
\left(\phi(\lambda) \rightarrow k\left(\lambda^{\prime}\right)\right)=\left(k(\lambda) \rightarrow k\left(\lambda^{\prime}\right)\right) .
$$

The inequality $\geq$ holds just because $\phi \leq k$ by Proposition 2. For the other inequality, assume an $a$ in $A$ satisfies $a \leq\left(\phi(\lambda) \rightarrow k\left(\lambda^{\prime}\right)\right)$, or equivalently $a \wedge \phi(\lambda) \leq k\left(\lambda^{\prime}\right)$. If $\mu$ denotes the left adjoint of $k$, we thus have $\mu(a \wedge \phi(\lambda)) \leq$ $\lambda^{\prime}$, hence by the Frobenius identity (Proposition 3), we have $\mu(a) \wedge \lambda \leq \lambda^{\prime}$, hence $\mu(a) \leq\left(\lambda \rightarrow \lambda^{\prime}\right)$, and hence by adjointness $a \leq k\left(\lambda \rightarrow \lambda^{\prime}\right)$. But since $k$ preserves $\wedge$, we have $k\left(\lambda \rightarrow \lambda^{\prime}\right) \leq k(\lambda) \rightarrow k\left(\lambda^{\prime}\right)$. So we conclude $a \leq k(\lambda) \rightarrow k\left(\lambda^{\prime}\right)$, and since this holds for all $a$, we get the other inequality $\leq$, and the Proposition is proved.

In the (Lawvere) conceptual framework mentioned in the introduction, the frame $O(M)$ associated to a locale $M$ may be thought of as an "algebra" of intensive quantities on the "space" $M$ (in particular, it behaves contravariantly with respect to locale maps $M \rightarrow N)$. One then gets a space $\mathcal{D}^{\prime}(\mathcal{M})$ of extensive quantities (behaving covariantly) on $M$ by taking the dual of $O(M)$ in an appropriate "linear" category, which we (in the spirit of [6]) take to be the category sl of sup-lattices, with $\Omega$ as the dualizing object. This general viewpoint was advocated by Lawvere in [9], [10], and studied in several special cases by Bunge, and in the particular one here, by Bunge and Funk, [1]. We follow them in thinking of $O^{\prime}(M)$ as consisting of a kind of "distributions" on $M$. So by definition,

$$
O^{\prime}(M)=\operatorname{sl}(O(M), \Omega),
$$


and this set inherits a (pointwise) sup-lattice structure from that of $\Omega$ (because the theory of sup-lattices is "commutative" in an appropriate sense). In general, $O^{\prime}(M)$ will neither be a frame nor a coframe. Now every sup lattice map $\mu: A \rightarrow \Omega$ has a right adjoint $k: \Omega \rightarrow A$, and this gives rise to an order-isomorphism $\operatorname{sl}(O(M), \Omega) \cong \mathrm{il}(\Omega, O(M))^{o p}$, where $\mathrm{il}(-,-)$ denotes the partially ordered set of inf -lattice maps. Also, by the standard correspondence between nuclei on $O(M)$ and sublocales of $M$, we have an isomorphism $\operatorname{sub}(M) \cong N(O(M))^{o p}$. Let us denote by sub. $(M)$ the lattice of those sublocales $M^{\prime} \subseteq M$ with the property that $M^{\prime} \rightarrow 1$ is open, i.e. is such that the unique frame map $\phi: \Omega \rightarrow O\left(M^{\prime}\right)$ has a left adjoint. This is easily seen to be equivalent to saying that the map $j \circ \phi: \Omega \rightarrow O(M)$ has a left adjoint (or equivalently, preserves infima), where $j$ is the nucleus corresponding to $M^{\prime} \subseteq M$. Let us call the lattice of these nuclei $N_{\bullet}(O(M))$. So the $\phi$ construction of Section 1 restricts to a map: $\Phi: N_{\bullet}(O(M)) \rightarrow \operatorname{il}(\Omega, O(M))$. Also, the construction $\Psi$ (restricted to il $(\Omega, O(M))$ ) factors through $N_{\bullet}$; for, if $k: \Omega \rightarrow O(M)$ is inf-preserving, it is a fixpoint for $\Phi \circ \Psi$ by Proposition 4 , meaning that $k=\Psi(k) \circ \phi$, so $\Psi(k) \circ \phi$ is inf-preserving since $k$ is. It follows that the adjoint pair $\Psi \dashv \Phi$ of Section 1 restricts to a pair of adjoints between il $(\Omega, O(M))$ and $N_{\bullet}(O(M))$.

Putting these together, we get a pair of adjoints

$$
\operatorname{sl}(O(M), \Omega) \cong \mathrm{il}(\Omega, O(M))^{o p} \underset{\Psi^{o p}}{\stackrel{\Phi^{o p}}{\leftrightarrows}} N_{\bullet}(O(M))^{o p} \cong \operatorname{sub}_{\bullet}(M)
$$

Since inf-lattice maps $k$ are fixed under $\Phi \circ \Psi$, as we just observed, it follows that $\operatorname{sl}(O(M), \Omega)$ is fixed under the adjoint pair displayed. Since the fixpoints for the composite $\Psi \circ \Phi$ are the relatively closed nuclei, it follows that the fixpoints on the right in the displayed pair of adjoints are those relatively closed sublocales $M^{\prime} \subseteq M$ which furthermore belong to sub. $(M)$, i.e such that $M^{\prime} \rightarrow 1$ are open. This is the Bunge-Funk correspondence, [1] Theorem 2.1. One may think of the $M^{\prime} \subseteq M$ corresponding to a frame distribution $\mu: O(M) \rightarrow \Omega$ as its support. 


\section{Regularization}

In this section we extend to open locales $M$ the well known topological interpretation of the double negation operator on opens of a topological space $M$ : $\neg \neg U=$ "interior of closure". The operator "interior of closure", or equivalently $\neg \neg$, applied to $U$, is called the regularization of $U$. The regular opens of $M$ constitute (classically) a complete Boolean algebra, $\operatorname{Reg}(M)$, which is not spatial, in general. In our context, closure and "negation" will be replaced by relative notions.

Let $M$ be a locale and let $o: O(M) \rightarrow \operatorname{Sub}(M)$ be the natural inclusion which to an $U \in O(M)$ associates the open sublocale $o(U)$ of $M$ given by the nucleus $U \rightarrow-$. Since $o$ preserves suprema, it has a right adjoint $o \vdash$ interior, as is well known. (We shall give an explicit formula for it in Lemma 1 below, but this formula is not needed in the proof of the main Theorem 2.)

We shall consider the map

$$
\int: O(M) \rightarrow O^{\prime}(M)
$$

given by $\left(\int U\right)(V)=\operatorname{pos}(U \cap V)$, where pos is left adjoint to the unique frame map $\phi: \Omega \rightarrow O(M)$; the existence of such left adjoint is the open-ness assumption on $M .{ }^{2}$ Since also $U \cap-$ is a left adjoint, $U \cap-\dashv(U \rightarrow-)$, it follows that

$$
\int U \dashv(U \rightarrow-) \circ \phi
$$

so in particular, $\int U$ is sup preserving, so $\int U \in O^{\prime}(M)$. The reader may think of $\int U$ as $\int_{U}$, i.e. as $f \mapsto \int_{U} f d A$.

Proposition 5 The map $\int: O(M) \rightarrow O^{\prime}(M)$ is a sup lattice map which preserves 1.

Proof. Preservation of suprema follows from the fact that suprema in $O^{\prime}(M)=\operatorname{sl}(O(M), \Omega)$ are computed pointwise. The last assertion is that $\int(M)$ is the largest frame distribution. But since $\int(M)=$ pos, this means that for any frame distribution $\mu$, we have $\mu \leq$ pos. By considering the right

\footnotetext{
${ }^{2}$ Our $\int$ is a restriction of the $\operatorname{map} \chi: \operatorname{sub}(M) \rightarrow \operatorname{sl}(O(M), \operatorname{sub}(1))$ considered in [1] under the name 'support map'. In [2], they analyze it in terms of the multiplicative action of intensive quantities on extensive ones, the "intensive" $u \in O(M)$ being sent to $u \cdot e_{!}$, where $e_{!}$is the distribution "total", which is synonymous with our pos.
} 
adjoints $k$ and $\phi$ of $\mu$ and pos, respectively, this is equivalent to $\phi \leq k$. But $\phi$ is the smallest map preserving the top element, by Proposition 2 .

Corollary 1 The map $\int: O(M) \rightarrow O^{\prime}(M)$ has a right adjoint $i$.

The map $i$ should be thought of as some kind of density-function formation, cf. also Bunge and Funk's [2] (their Proposition 3.4 seems to be analogous to part of the following). We don't have an explicit formula for $i$, but we do have one for its composite with $\int$ in the following Theorem. Recall that the fixpoints for $\Psi^{o p} \circ \Phi^{o p}: S u b(M) \rightarrow S u b(M)$ are the relatively closed sublocales, and in fact is is clear from the adjointness between $\Phi$ and $\Psi$ that this composite is in fact the relative closure formation. We shall denote it closure. Then we have

Theorem 2 The following operators on $O(M)$ coincide:

(i) $i \circ \int(-)$;

(ii) $\bigwedge_{\lambda \in \Omega}(-\rightarrow \phi(\lambda)) \rightarrow \phi(\lambda)$ (which we shall denote $L_{\Omega}(-)$ );

(iii) interior o closure $\circ$ o.

In particular, this operator is a nucleus on $O(M)$.

Proof. We investigate the effect of these three operations on a fixed open sublocale $V$. For short, when we say that we "prove (i) $\leq$ (ii)", we mean that we prove that the result of applying the operator in (i) to $V$ is $\leq$ the result of applying (ii) to $V$.

To show that $(\mathrm{i})=(\mathrm{ii})$, we first prove the inequality $(\mathrm{i}) \leq(\mathrm{ii})$. So assume $U \in O(M)$ satisfies $U \leq i\left(\int V\right)$. We need to show $U \leq \wedge(V \rightarrow \phi(\lambda)) \rightarrow \phi(\lambda)$. But

$$
U \leq i\left(\int V\right)
$$

if and only if

$$
\int U \leq \int V
$$

if and only if

$$
V \rightarrow \phi(-) \leq U \rightarrow \phi(-)
$$


(by "mating", using (3)) which in turn holds if and only if

$$
V \rightarrow \phi(\lambda) \leq U \rightarrow \phi(\lambda) \text { for all } \lambda \in \Omega .
$$

This in turn implies the second inequality in

$$
U \leq(U \rightarrow \phi(\lambda)) \rightarrow \phi(\lambda) \leq(V \rightarrow \phi(\lambda)) \rightarrow \phi(\lambda)
$$

so that

$$
U \leq \bigwedge_{\lambda \in \Omega}(V \rightarrow \phi(\lambda)) \rightarrow \phi(\lambda)
$$

Conversely, to prove (ii) $\leq(\mathrm{i})$, assume that $U \leq \wedge_{\lambda \in \Omega}(V \rightarrow \phi(\lambda)) \rightarrow \phi(\lambda)$. We need to show $U \leq i\left(\int V\right)$. We have for all $\lambda \in \Omega$ that $U \leq(V \rightarrow \phi(\lambda)) \rightarrow$ $\phi(\lambda)$ and hence also

$$
(U \rightarrow \phi(\lambda)) \rightarrow \phi(\lambda) \leq(V \rightarrow \phi(\lambda)) \rightarrow \phi(\lambda)
$$

since $(-\rightarrow \phi(\lambda)) \rightarrow \phi(\lambda)$ is a closure operator. Applying $-\rightarrow \phi(\lambda)$, we get

$$
((V \rightarrow \phi(\lambda)) \rightarrow \phi(\lambda)) \rightarrow \phi(\lambda) \leq((U \rightarrow \phi(\lambda)) \rightarrow \phi(\lambda)) \rightarrow \phi(\lambda),
$$

and using the law analogous to $\neg \neg \neg=\neg$, we conclude that $V \rightarrow \phi(\lambda) \leq$ $U \rightarrow \phi(\lambda)$; this holds for all $\lambda \in \Omega$, so

$$
V \rightarrow \phi(-) \leq U \rightarrow \phi(-) .
$$

By mating and the formula (3), we get $\int U \leq \int V$, or equivalently $U \leq$ $i\left(\int V\right)$.

To prove that $(\mathrm{i})=(\mathrm{iii})$, we note the following reformulation of formula $(3)$ :

Proposition 6 We have $\int=\Phi^{o p} \circ o: O(M) \rightarrow O^{\prime}(M)$ and $i=$ interior $\circ$ $\Psi^{o p}: O^{\prime}(M) \rightarrow O(M)$.

Proof. The right adjoint of $\int U$ is given by formula (3), thus by $\Phi(U \rightarrow$ -) which is also by definition the right adjoint of $\Phi^{o p}(o(U))$. The second formula follows from the first by taking right adjoints.

Composing the last formula on the right with $\int$ gives

$i \circ \int=$ interior $\circ \Psi^{o p} \circ \int=$ interior $\circ \Psi^{o p} \circ \Phi^{o p} \circ o=$ interior $\circ$ closure $\circ$ o. 
This proves that the three operators agree. Since the second one is a (generalized double negation) nucleus, the last assertion follows.

Generalized double negation nuclei appear also in [5] and [8]. The $L_{\Omega}$ of the Theorem will be denoted just by $L$ in the following. So $M_{L}$ denotes the locale whose frame of opens is the frame of fixpoints for $L$. (They are the "relatively regular opens".) It is a sublocale of $M$, and in fact, according to [5] Lemma 1.2, is the smallest dense sublocale of $M$, for the appropriate relative notion of density. The following result is an immediate consequence of results in loc. cit. (notably Lemma 1.11 (ii)), but we shall give a direct proof, not utilizing these:

Proposition 7 If $M$ is an open locale, then so is the locale $M_{L}$

Proof. It is immediate that every $\phi(\lambda)$ is fixed for $L$, so that $\phi: \Omega \rightarrow$ $O(M)$ factors across the inclusion $I: \operatorname{Fix}(L) \rightarrow O(M)$, by an order preserving map $\phi^{\prime}$, say. Since $I$ is full and faithful and has adjoints on both sides, it follows that $\phi^{\prime}$ has adjoints on both sides. In fact, pos $\circ I$ is a left adjoint of $\phi^{\prime}$, and thus $M_{L}$ is open (Frobenius identity being automatic for left adjoints into $\Omega$, by Proposition 3 ).

Proposition 8 The construction $M \mapsto M_{L}$ defined on open locales is idempotent. Locales of form $M_{L}$ are precisely the open pre-boolean locales, in the sense of [8].

Proof. The first assertion may be deduced from Johnstone's density characterization of the $M_{L}$ construction, as quoted above, together with the fact that a composite of two dense maps is dense. A more elementary proof goes as follows. We want to prove that the "generalized double negation" nucleus $L^{\prime}$ for the frame $\operatorname{Fix}(L)$ is the identity. But the inclusion $\operatorname{Fix}(L) \subseteq$ $O(M)$ preserves meets (being a right adjoint) and also preserves $\rightarrow$; and finally, since the elements of form $\phi(\lambda) \in O(M)$ also are fixed for $L, \phi$ factors through $\operatorname{Fix}(L)$ by a frame map $\phi^{\prime}: \Omega \rightarrow \operatorname{Fix}(L)$, and thus all the building blocks for $L^{\prime}$ are preserved by the inclusion $\operatorname{Fix}(L) \subseteq O(M)$, and thus $L$ on $O(M)$ restricts to $L^{\prime}$ on $\operatorname{Fix}(L)$. Since $L$ is certainly the identity operator on $\operatorname{Fix}(L)$, it follows that so is $L^{\prime}$, proving the first assertion. The second assertion follows from the openness assertion of the previous Proposition. 
We finish by a generalization of the equality (ii)=(iii) of Theorem 2 . Let $X$ be an arbitrary subset of a frame $O(M)$. If $j$ is a nucleus on $O(M)$, we denote by closure $_{X}(j)$ the closure of $j$ with respect to $X$, which by Proposition 1 is given as $\bigvee_{x} c(j(x)) \wedge o(x)$, the smallest nucleus agreeing with $j$ on $X$. (It is $\leq j$ in the frame $N(O(M))$; the word "closure" refers to the dual lattice $\operatorname{Sub}(M)$.)

Recall also that $o: O(M) \rightarrow \operatorname{Sub}(M)$ has a right adjoint interior : $\operatorname{Sub}(M) \rightarrow O(M)$. We then have

Theorem 3 For any set $X \subseteq O(M)$, we have

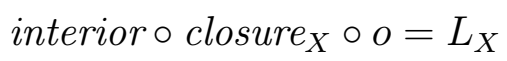

where $L_{X}$ is the generalized double negation nucleus

$$
\bigwedge_{x \in X}(-\rightarrow x) \rightarrow x
$$

Proof. Recall that $N(O(M))$ under the pointwise ordering is a frame, in particular a Heyting algebra, so that $\neg$ makes sense in it. We shall need

Lemma 1 Given a nucleus $j$, then interior $(j) \in O(M)$ is the element $(\neg j)(0)$.

Proof. This will follow by proving that it satisfies the two adjunction inequalities characterizing the right adjoint of $o$. They are

$$
y \leq(\neg O(y))(0) \text { for all } y \text { in } O(M)
$$

and an inequality in $\operatorname{Sub}(M)$, which in the dual poset $N(O(M))$ is

$$
j \leq o((\neg j)(0)) \text { for all } j \text { in } N(O(M)),
$$

respectively. The former is actually an equality: the open nucleus $o(y)$ has a complement, namely the closed nucleus $c(y)=y \vee-$, which is thus $\neg o(y)$, and certainly $c(y)(0)=y$. The second inequality means that

$$
j(y) \leq((\neg j)(0)) \rightarrow y
$$


for all $y$. But

$$
j(y) \wedge(\neg j)(0) \leq j(y) \wedge(\neg j)(y)=(j \wedge \neg j)(y)=y
$$

since meets are computed pointwise, and the bottom nucleus $j \wedge \neg j$ is the identity map of $O(M)$. From this, the desired inequality follows by exponential adjointness, and the Lemma is proved.

We shall need yet another calculation.

If $x, y, z$ are elements in any Heyting algebra $A$, with $x \leq z$, then

$$
z \rightarrow(x \vee(z \rightarrow(x \vee y))) \leq z \rightarrow(x \vee y)
$$

For

$$
\begin{array}{rlrl} 
& (z \rightarrow(x \vee(z \rightarrow(x \vee y)))) \wedge z & \\
\leq & (x \vee(z \rightarrow(x \vee y)) \wedge z & & \text { by modus ponens } \\
= & (x \wedge z) \vee(z \rightarrow(x \vee y)) \wedge z & & \text { by distributivity } \\
= & x \vee(z \rightarrow(x \vee y)) \wedge z & & \text { since } x \leq z \\
\leq & x \vee(x \vee y)=x \vee y & \text { by modus ponens, }
\end{array}
$$

so by exponential adjointness, we get (4).

If now $A$ is a frame $O(M)$, and $x \leq z$, the inequality (4) implies that the composite $o(z) \circ c(x)$ of the nuclei $c(x)=x \vee-$ and $o(z)=(z \rightarrow-)$ is an idempotent map. But it is well known, and easy to see, that the composite $j_{1} \circ j_{2}$ of two nuclei is a nucleus iff it is idempotent, in which case $j_{1} \circ j_{2}=j_{1} \vee j_{2}$ in the frame of nuclei. It follows that for $x \leq z, o(z) \circ c(x)$ is a nucleus and is the join $o(z) \vee c(x)$ in the frame of nuclei. (Nuclei of this form are exactly those that correspond to locally closed sublocales of $M$, cf. e.g. [7].) Applying this to the case where $z=u \rightarrow x$, we conclude in particular that

$$
\begin{aligned}
& (o(u \rightarrow x) \vee c(x))(0)=(o(u \rightarrow x) \circ c(x))(0)= \\
= & o(u \rightarrow x)(x \vee 0)=o(u \rightarrow x)(x)=(u \rightarrow x) \rightarrow x .
\end{aligned}
$$

Therefore

$$
\bigwedge_{x}(u \rightarrow x) \rightarrow x=\bigwedge_{x}(o(u \rightarrow x) \vee c(x))(0)
$$

(meets of nuclei being computed pointwise). On the other hand, Proposition 1 applied to the nucleus $o(u)$ gives

$$
\text { closure }_{X}(o(u))=\bigvee_{x} c(u \rightarrow x) \wedge o(x)
$$


(a non-pointwise sup of nuclei), so

$$
\neg \operatorname{closure}_{X}(o(u))=\bigwedge_{x} o(u \rightarrow x) \vee c(x),
$$

and hence, by (5), we conclude that

$$
\neg \text { closure }_{X}(o(u))(0)=\bigwedge_{x}(u \rightarrow x) \rightarrow x .
$$

By the Lemma, the left hand side here is the interior of the $X$-closure of $o(u)$, proving the Theorem.

\section{References}

[1] M. Bunge and J. Funk, Constructive Theory of the Lower Power Locale, Math. Struct. in Comp. Science 6 (1996), 69-83.

[2] M. Bunge and J. Funk, Spreads and the symmetric topos II, McGill Report 96-04!996

[3] M. Jibladze and P.T. Johnstone, The frame of fibrewise closed nuclei, Cahiers Top. Géom. Diff. Catégoriques 32 (1991), 99-112.

[4] P.T. Johnstone, Stone Spaces, Cambridge University Press 1982

[5] P.T. Johnstone, A constructive 'closed subgroup theorem' for localic groups and groupoids, Cahiers Top. Géom. Diff. Catégoriques 30 (1989), $3-23$.

[6] A. Joyal and M. Tierney, An extension of the Galois theory of Grothendieck, Memoirs A.M.S. 309 (1984)

[7] A. Kock and T. Plewe, Glueing analysis for complemented subtoposes, Theory and Appl. of Categories Vol. 2 No. 9 (1996).

[8] A. Kock and G.E. Reyes, Relatively Boolean and de Morgan Toposes and Locales, Cahiers Top. Géom. Diff. Catégoriques 35 (1994), 249-261.

[9] F.W. Lawvere, Measures on Toposes, Lectures given at the Workshop on Category Theoretic Methods in Geometry, Aarhus University 1983 (unpublished). 
[10] F.W. Lawvere, Categories of space and quantity. In J. Echeverría, A. Ibarra, and T. Mormann (eds.). The Space of Mathematics. Berlin: de Gruyter 1992

Aarhus Universitet and Université de Montréal, December 1997 


\section{Recent BRICS Report Series Publications}

RS-97-33 Anders Kock and Gonzalo E. Reyes. A Note on Frame Distributions. December 1997. 15 pp.

RS-97-32 Thore Husfeldt and Theis Rauhe. Hardness Results for Dynamic Problems by Extensions of Fredman and Saks' Chronogram Method. November 1997. i+13 pp.

RS-97-31 Klaus Havelund, Arne Skou, Kim G. Larsen, and Kristian Lund. Formal Modeling and Analysis of an Audio/Video Protocol: An Industrial Case Study Using UPPAAL. November 1997. 23 pp. To appear in The 18th IEEE Real-Time Systems Symposium, RTSS '97 Proceedings.

RS-97-30 Ulrich Kohlenbach. Proof Theory and Computational Analysis. November 1997. 38 pp.

RS-97-29 Luca Aceto, Augusto Burgueño, and Kim G. Larsen. Model Checking via Reachability Testing for Timed Automata. November 1997. 29 pp.

RS-97-28 Ronald Cramer, Ivan B. Damgård, and Ueli Maurer. Span Programs and General Secure Multi-Party Computation. November 1997. 27 pp.

RS-97-27 Ronald Cramer and Ivan B. Damgård. Zero-Knowledge Proofs for Finite Field Arithmetic or: Can Zero-Knowledge be for Free? November 1997. 33 pp.

RS-97-26 Luca Aceto and Anna Ingólfsdóttir. A Characterization of Finitary Bisimulation. October 1997. 9 pp. To appear in Information Processing Letters.

RS-97-25 David A. Mix Barrington, Chi-Jen Lu, Peter Bro Miltersen, and Sven Skyum. Searching Constant Width Mazes Captures the $A C^{0}$ Hierarchy. September 1997. 20 pp. To appear in STACS '98: 15th Annual Symposium on Theoretical Aspects of Computer Science Proceedings, LNCS, 1998.

RS-97-24 Søren B. Lassen. Relational Reasoning about Contexts. September 1997. 45 pp. To appear as a chapter in the book Higher Order Operational Techniques in Semantics, eds. Andrew D. Gordon and Andrew M. Pitts, Cambridge University Press. 